\title{
El currículum tras las rejas: aproximaciones al significado pedagógico-curricular en un contexto privativo de libertad para adolescentes
}

\author{
Alba Sepúlveda \\ Pontificia Universidad Católica de Chile
}

\begin{abstract}
Resumen
Este trabajo analiza la implementación del Marco Curricular de Adultos en la educación de las y los jóvenes que se encuentran en un contexto privativo de libertad. Para esto se hace una revisión de diversos documentos legales y estudios que contextualizan la implementación de este curriculum en la educación de los jóvenes recluidos, a través del uso de la técnica de análisis de contenido. Estos hallazgos se contrastan a la luz de los antecedentes y referentes teóricos y se plantean como los ejes de discusión y conclusiones. El trabajo identifica importantes problemas sobre los silencios de diversas legislaciones que hoy en día regulan la educación de los jóvenes privados de libertad. Estos vacíos legales podrían incidir en la utilización arbitraria del currículo de educación para adultos en la educación de jóvenes privados de libertad.
\end{abstract}

Palabras clave: Curriculum de Adultos, jóvenes privados de libertad, educación penal.

The curriculum in jail: an aproache to the pedagogical-curricular meaning in a context of privative freedom for teenagers

\begin{abstract}
This paper analyzes the implementation of the Adult Curricular Framework for incarcerated youth. It reviews diverse legal documents and studies that contextualize the enactment of this curriculum within incarcerated education. Through the application of content analysis to the adult curriculum. The paper identifies relevant problems about the silences of different laws that regulate the education of young inmates today. These loopholes may impact in the arbitrary use of the adult curriculum for incarcerate youth.
\end{abstract}

Keywords: Adult curriculum, youth private of liberty, penal education.

\section{El currículum tras las rejas}

La presente investigación es el resultado de un proceso de revisión y análisis teórico, sobre el Marco Curricular de educación de Adultos, que se implementa en la educación de las y los jóvenes que se encuentran en un contexto privativo de libertad para menores de edad. En este sentido, su objeto de estudio es el curriculum de adultos, en tanto escenario posible para revisar y analizar la trama de significaciones o ideas que le subyacen y configuran en relación con perspectivas pedagógico-curriculares críticas y legales-educativas.

\footnotetext{
*Dirección de correspondencia [Correspondence address]:

Alba Sepúlveda, Pontificia Universidad Católica de Chile E-mail: alsepulveda@uc.cl
}

La relevancia de esta investigación está dada por la necesidad de comprender las decisiones, ideas, concepciones y supuestos que sustentan la implementación del curriculum de adultos en la educación de los jóvenes que permanecen en dicho contexto. En este sentido, la comprensión de esta problemática pedagógica-curricular podría contribuir a la revisión de las políticas educativas que regulan actualmente en el país, los procesos de educación formal, en este caso, la concerniente a la modalidad de adultos, que incluye como sujeto de aprendizaje al adulto, pero que se implementa en un porcentaje considerable de jóvenes que se encuentran en el sistema de justicia juvenil.

En lo que respecta al campo penal-educativo, la indagación podría contribuir a revisar de manera exhaustiva la concepción e implementación de la Ley de Responsabilidad Penal Adolescente, en cuanto a la noción de "intervención socio-educativa 
amplia", en la que no se define o señala las orientaciones pedagógicas-curriculares que serían favorables desarrollar en el marco de un contexto privativo de libertad y que pueden contribuir a generar escenarios de formación y ejercicio de los valores o principios fundamentales.

Asimismo, contribuye a develar la vigencia de un marco jurídico que se mantiene desde hace más de 30 años, bajo una noción que señala a los sujetos que infringen la ley como irregulares sociales, y que establece, a través de la alianza entre el Ministerio de Justicia y Ministerio de Educación, una labor educativa conjunta orientada a lograr la rehabilitación de los irregulares sociales y de conducta, internados en los establecimientos penales del país (Congreso Nacional de Chile, 1978). En este sentido, el trabajo podría contribuir a mejorar o resignificar de manera importante la legislación que desde 1978 ha sido el sustento para la concepción e implementación de la educación en contextos privativos de libertad.

Concerniente al campo académico, la indagación podría favorecer la reflexión y debate respecto de la concepción de la educación como medida de prevención especial, cuando tiende a compatibilizarse con la sanción y termina asociándose con un tratamiento correctivo o un castigo, cuyo propósito es la corrección del joven, más no la prevención del delito, finalidad planteada por las legislaciones internacionales como las Directrices para la prevención de la delincuencia juvenil y las Normas para la protección de menores de edad privados de libertad (Organización de las Naciones Unidas, 2007).

Del mismo modo, el trabajo posibilita abrir la discusión académica dentro de las investigaciones en el ámbito curricular, sobre la pertinencia de formar sujetos de educación para adultos a los jóvenes que se encuentran en contextos privativos de libertad.

En cuanto al Servicio Nacional de Menores, el presente trabajo aportaría en la consolidación de una efectiva estrategia de protección y garantía al derecho a una educación pertinente y de calidad para la población juvenil que se encuentra en condición de reclusión.

Bajo este panorama, se plantean las siguientes preguntas de investigación: ¿Por qué el marco curricular de adultos es la orientación que regula la educación de los jóvenes que se encuentran en contextos privativos de libertad?; ¿Cuál es el propósito de este currículum en un contexto privativo de libertad? y ¿Es ésta la propuesta curricular más pertinente para la educación de los jóvenes?

El objetivo de investigación es analizar la trama de significaciones e ideologías tácitas en las políticas, estudios, programas o investigaciones en las que se sustenta el marco curricular de adultos que se implementa en un contexto privativo de libertad para menores de edad.

\section{La educación de los jóvenes privados de li- bertad}

La Convención sobre los Derechos del Niño, proclamada por la Asamblea General de las Naciones Unidas en 1989, se presenta como la estructura jurídica que legitima los derechos de los niños/as, como ley internacional, reconociendo como sujeto de derecho a las personas menores de 18 años de la misma forma que los adultos.

Del mismo modo, la convención considera que el niño debe estar plenamente preparado para una vida independiente en sociedad y ser educado en el fomento de un espíritu de paz, dignidad, tolerancia, libertad, igualdad y solidaridad (UNICEF, 1996).

En lo que respecta a la educación, la Convención declara que los Estados partes reconocerán el derecho del niño a la educación en condiciones de igualdad de oportunidades. Por otra parte, las Directrices de las Naciones Unidas para la prevención de la delincuencia juvenil — Directrices Riad — señalan que "el joven debe desempeñar una función activa y participativa en la sociedad y no deben ser considerados meros objetos de socialización o control" (Organización de las Naciones Unidas, 2007:78). A su vez, presenta tres características principales: alcance, enfoque proactivo de la prevención y la concepción de los niños como miembros de pleno derecho en la sociedad. Estos principios, se conciben como fundamentales en la promoción, formación de valores y potenciación de una convivencia basada en el ejercicio de los derechos humanos.

Paralelamente a las Directrices Riad, las reglas para la protección de los menores de edad privados de libertad, establecen las normas mínimas aceptadas por las Naciones Unidas sobre la protección de los menores de edad en esta condición, las cuales son compatibles con los derechos humanos y tienen el propósito de contrarrestar los efectos nocivos de la reclusión y favorecer la integración del joven en la sociedad. En el ámbito de la educación formal, la formación profesional y el trabajo, todo menor "tendrá derecho a recibir una enseñanza adaptada a sus necesidades, capacidades y destinada a prepararlo para su reinserción en la sociedad" (Organización de las Naciones Unidas, 2007:95). Lo anterior, debe complementarse con el derecho a recibir una formación que le permita proseguir con estudios superiores y poder ejercer una profesión para 
un futuro empleo.

Valenzuela, en su estudio "La Pena y la Educación" (2009), ofrece una aproximación al fundamento de la pena juvenil que la hace divergente de la pena de adultos. Sobre la pena de menores de edad, plantea que uno de los aspectos centrales en la práctica punitiva, en cuanto sanción que se le asigna al autor de un delito, es la educación. Sin embargo, en esta relación que necesariamente se da cuando se ejecuta una pena, la educación tiende a compatibilizarse y asociarse con la medida de castigo. Valenzuela (2009), critica esta combinación, señalando que "si la educación es el fundamento de la práctica punitiva general y está asociada con la justificación del castigo, la pena pierde reconocimiento como tal, pues la educación tendría el atributo de sufrimiento característico del castigo" (186).

Por su parte, Couso (2007), plantea que en el derecho penal adolescente se suele partir de la idea que el principio educativo o medida preventiva-especial juega un papel central en la imposición, determinación y ejecución de la pena, convirtiéndose en lo que diferencia la pena de adultos de la de menores de edad.

Bajo esta perspectiva, la educación no plantea una finalidad liberadora, participativa y de desarrollo de la personalidad del joven, que cuente con su autonomía y considere su subjetividad. Así, la educación como medida preventiva requiere y cuenta con el contexto coactivo de la justicia penal, por lo cual sanción-educación terminan combinándose para atender a finalidades de resocialización, reinserción y rehabilitación (Couso, 2007).

Sobre esta misma línea, el modelo de responsabilidad actual contenido en la Ley 20.084 (Ministerio de Justicia, 2005), según Aguirrezabal, Lagos, y Vargas (2009), se sustenta a partir de dos consideraciones fundamentales suscitadas en la Convención de los Derechos del Niño (UNICEF, 1996). Una corresponde a que el niño no es menos relevante que el adulto, es también un ser humano que se encuentra en la primera etapa de desarrollo, que por falta de madurez física y mental necesita de cuidados especiales y debida protección legal. La otra consideración, refiere a que la infancia y la adolescencia son etapas del desarrollo humano que tienen igual valor en cualquier periodo de la existencia. Para la convención de los derechos del niño, se entiende por niño todo ser humano menor de 18 años (1996).

Por consiguiente, según las autoras, en la pena de menores de edad se limita al mínimo indispensable la intervención de la justicia penal, con una serie de sanciones como respuesta jurídica al delito. Estas sanciones están basadas en principios educa- tivos de readaptación y resocialización, que se encuentran vigentes en la determinación y ejecución de las sanciones y se implementan como una medida preventiva-especial mixta entre responsabilidadmerecimiento, en términos de readaptación o reinserción social y educación, y en términos de acción constructiva de la personalidad del joven para su vida en sociedad.

Por otra parte, la organización penal introduce la idea de garantizar una pedagogía universal del trabajo, la que permitiría en el individuo perezoso, la afición al mismo, obligándolo a colocarse en un sistema de intereses en el que el trabajo será más ventajoso que la pereza y formará en torno suyo la idea de "obligación al trabajo, pero también retribución que permita al preso mejorar su suerte durante el periodo de detención y después de él" (Foucault, 2009:114). Bajo esta idea, podrían ser corregidas sus costumbres, pues un individuo habituado a trabajar ha aprendido un oficio que le garantiza su subsistencia sin peligro.

\section{Perspectiva Crítica ${ }^{1}$ del Curriculum en tanto campo de experiencia}

La perspectiva crítica comprende el curriculum como un campo de experiencia, poder, interrogación y cuestionamiento. En este sentido, aporta elementos de análisis relacionados con la correspondencia entre las relaciones sociales de la escuela y las relaciones sociales del puesto de trabajo; los valores, normas y disposiciones que se fijan como necesarias para un buen funcionamiento de la sociedad; y la escuela y el curriculum como esfera pública democrática.

Estas cuestiones son claves para orientar el análisis y la comprensión de los presupuestos e ideologías tácitas que describen el curriculum, así como para relevar su sentido, su función social y sus significados con respecto a que éste no puede alejarse de la

\footnotetext{
${ }^{1}$ La perspectiva crítica del currículum, proviene de los años 60, una década marcada por movimientos de independencia y reivindicación de Derechos, en la cual surgen teorizaciones que cuestionan el sistema educacional tradicional establecido. Según Da Silva (1999), "para la literatura educacional estadounidense, la renovación de la teoría sobre currículo parece haber sido exclusividad del llamado movimiento de reconceptualización. De la misma forma, la literatura inglesa reivindica prioridad para la llamada nueva sociología de la educación, un movimiento identificado con el sociólogo inglés Michael Young. Una revisión brasilera no dejaría de señalar el importante papel de la obra de Paulo Freire, en cuanto a los franceses ciertamente no dejarían de destacar el papel de los ensayos de Althusser, Bourdieu y Passeron, Baudelot y Establet" (13). Las teorías críticas sobre el currículo, comienzan por poner acento en la relación de la educación establecida, con las relaciones de poder, desigualdad e injusticia social. En este sentido, interrogan el Status quo.
} 
realidad social que lo configura, considerando atenta y seriamente las necesidades, intereses y expectativas de la comunidad educativa, siendo imprescindible reducir la brecha entre lo que sucede en el escenario educativo y lo que se estructura y legitima en la sociedad.

Un primer elemento de la perspectiva crítica del currículum, tiene relación con la correspondencia entre las relaciones sociales de la escuela y las relaciones sociales del puesto de trabajo. Al respecto, Bowles y Gintis (1976) señalan que la concepción de aprendizaje que está inmersa en el curriculum, puede ser determinante en la reproducción y justificación de las creencias explícitas de la deseabilidad de las estructuras sociales existentes. Por ejemplo, un curriculum cuyas metas de aprendizaje enfaticen el desarrollo de actitudes y habilidades como la obediencia a la norma, el seguimiento de instrucciones, la puntualidad y constancia, las cuales forman parte de los aprendizajes que garantizan la inserción efectiva al mundo laboral y productivo; es un curriculum que mantiene una correspondencia entre las relaciones sociales que se desarrollan en la escuela y las relaciones sociales del puesto de trabajo.

De allí que las metas de aprendizaje sean un mecanismo de reafirmación y reproducción de las desigualdades entre una persona que obedece órdenes y otra que es autónoma y dirige instrucciones. En términos de los autores, "a través de las metas del aprendizaje se desarrollan las actitudes, habilidades y conocimientos necesarios para ser un buen trabajador" (Bowles y Gintis, 1976:15).

Sobre esta misma línea, Apple (1986) señala la necesidad imperativa en que el currículum escolar debe trascender de la definición, transmisión y reproducción de unos contenidos que el estudiante debe aprender y de relaciones sociales basadas en el poder, a un cuestionamiento de las condiciones presentes, que inciden en las prácticas de los actores educativos involucrados en el proceso enseñanza- aprendizaje, para que tanto ética, política, económica y socialmente puedan actuar comprometida y responsablemente sobre sus propias vidas y sobre las de los sujetos con los cuales interactúan en el sistema escolar. De lo contrario, plantea el autor, "sucederá esta inmersión preocupante en la tendencia de reproducción de los patrones culturales, sociales, económicos que ratifican las necesidades y desigualdades en los sujetos pertenecientes a los grupos menos favorecidos" (Apple, 1986:65).

La perspectiva crítica del curriculum, desde la mirada de Apple (1986), se interroga y cuestiona acerca de los valores, normas y disposiciones que se fijan como necesarias para el funcionamiento y desarrollo de la vida institucional y social. De esta manera, el curriculum juega un papel importante en la selección, organización, conservación y transmisión de estas normas y disposiciones, pues se convierte en un mecanismo para comunicar y transferir a la comunidad educativa, el conocimiento, las prácticas, los valores y las competencias que son importantes y necesarias, como aquellas que no lo son.

Sin embargo, la escuela y el curriculum existen, mediante su relación o nexo con otras instituciones, lo cual se constituye como un rasgo central en su función social, que incide en la determinación de las distintas poblaciones escolares (Apple, 1986:83).

Ello hace que la comunidad curricular sea esencial, entendiendo con esto, la confluencia de interacciones y acciones de todos los actores que se involucran en el proceso educativo, donde se origine una parte de la crítica sistemática y permanente de las ideologías, presupuestos y prácticas que ayudan a producir y potenciar la desigualdad y la perpetuación de los intereses sociales, económicos, culturales y políticos de ciertos grupos u organizaciones por encima de otros.

Esto significa que la construcción y el desarrollo del curriculum, debe adoptar una posición de análisis, reflexión y de defensa desde perspectivas críticas, tanto dentro como fuera del campo curricular. Entre las posturas internas más importantes estaría, según Apple (1986) "la de apoyar los derechos de los estudiantes (y los derechos democráticos de los profesores, grupos oprimidos y otros), dado que en el curriculum en cuanto que campo tiene como una de sus preocupaciones primordiales la tarea de crear accesos al conocimiento, libre acceso a una información política y culturalmente sincera y a la expresión pública, basada en que esta no se puede separar de nuestra búsqueda de entornos educativos justos" (90).

No obstante, esta postura de análisis y reflexión crítica en el curriculum, no solo debe guiar la defensa de acceso y ejercicio de los derechos económicos, legales y culturales de los profesores, estudiantes y demás actores de la comunidad educativa, sino detenerse y evaluar críticamente las finalidades de los modelos terapéuticos que se implementan en la educación, bajo la excusa de cambiar a un individuo sin tener en cuenta las condiciones y estructuras socioculturales de la escuela y del contexto en la que se encuentra inserta.

En virtud de las perspectivas críticas abordadas sobre el estudio del curriculum, el planteamiento de Giroux (1990) es particularmente ilustrativo, en cuanto a la concepción de la escuela y el curricu- 
lum como campo de acción o esfera pública democrática, el cual requiere ser interrogado con respecto a los presupuestos y principios subyacentes que lo configuran y determinan, desde la racionalidad tecnocrática, relacionados con "las pretensiones de verdad explícita, el conocimiento como neutral y objetivo y los juicios de valor separados de los hechos y modos de investigación que pueden y deben ser objetivos (53).

Esta pretensión de objetividad, avala la idea de que sean los grupos de expertos y sabios los que fijen los criterios normativos que definen el curriculum, "dejando de lado la examinación del modo en que las comunidades educativas legitiman ciertas formas de conocimiento e intereses culturales" (Giroux, 1990:57), reduciendo el escenario de poder, decisión y participación de profesores y estudiantes en la construcción y desarrollo del campo curricular. En términos de Apple (1986) impidiendo en que tanto ética, política, económica y socialmente profesores y estudiantes puedan actuar comprometida y responsablemente sobre sus propias vidas y sobre las de los sujetos con los cuales interactúan en el sistema escolar.

En contraposición a esta racionalidad tecnocrática, se presenta la nueva sociología del curriculum como una alternativa seria que "defiende con firmeza que las escuelas forman parte de un proceso societario más amplio y que por eso mismo deben juzgarse dentro de un contexto socio-económico específico" (Giroux, 1990:57). Sobre esta mirada, se respalda la necesidad de crear desde la participación de profesores, estudiantes y demás actores de la comunidad educativa, espacios de reexaminación y análisis, que les permita involucrarse activamente en las actividades de crítica en cuanto a las relaciones existentes entre curriculum, escuela y sociedad.

\section{Metodología}

El diseño de investigación a desarrollar es de corte cualitativo, puesto que como modo de investigación permite abordar de manera adecuada problemas concretos, mediante la expresión de peculiaridades de un contexto determinado (Ruiz Olabuénaga, 2003) dejando de lado la búsqueda por la universalidad y procurando la comprensión en profundidad de un fenómeno (Bericat, 1998).

Se aplicó la técnica de análisis de contenido al curriculum de adultos y al de educación básica y media regular ${ }^{2}$ como forma de recolección y análisis

\footnotetext{
${ }^{2}$ Para efectos de la indagación se toman los Objetivos Fundamentales y Contenidos Mínimos Obligatorios de la Educación básica y Media, actualización 2009.
}

de la información. Este consistió en la recolección y análisis de la información que contiene el curriculum de adultos, a través de una lectura rigurosa que permite identificar las nociones, concepciones, ideologías y significados que configuran dicho marco.

Se utilizó el enfoque narrativo, puesto que este tipo de análisis de contenido permite comprender la posición relativa de ciertos elementos descritos en el texto, considerando la existencia de redes que configuran los significados descritos en un contexto determinado.

Del curriculum de educación básica y media regular, se seleccionaron de igual manera las secciones que se tomaron en el de adultos, incluyendo los principios valóricos del curriculum y las fuentes de construcción curricular; y manteniendo solo los sectores de aprendizaje de lenguaje y comunicación y matemáticas.

Por otra parte, la intención en el análisis comparativo de los dos Marcos Curriculares, se sustenta en visibilizar e identificar las nociones, ideas o significados que se les atribuye a las categorías pedagógico-curriculares, más no, en evaluar la calidad y pertinencia del uno sobre el otro. En este caso, se incorporan categorías de análisis propias de la investigación cualitativa y junto con las categorías propias de la investigación educativa. Por ello, es importante puntualizar que se ha tomado como referente para elaborar este diseño planteamientos de autores como Sandín (2003), Valles (2000), Flick (2004), Ruiz (2003), entre otros.

Del curriculum de adultos se tomaron las secciones concernientes a: la normativa legal del curriculum, los requerimientos de diseño curricular, los pilares de construcción curricular, los principios que orientan el curriculum, la definición de la estructura curricular, las orientaciones de selección curricular, los Objetivos Fundamentales, los Objetivos Fundamentales Verticales y Transversales, los Contenidos Mínimos Obligatorios, los sectores y subsectores del aprendizaje, los ámbitos de formación, la formación instrumental de convivencia social, consumo y calidad de vida, inserción laboral, las tecnologías de información y comunicación, el sector de lenguaje y comunicación, matemáticas, ciencias, estudios sociales e idioma extranjero.

Del curriculum de educación básica y media regular, se seleccionaron de igual manera las secciones que se tomaron en el de adultos, incluyendo los principios valóricos del curriculum y las fuentes de construcción curricular; y manteniendo solo los sectores de aprendizaje de lenguaje y comunicación y matemáticas. 
Se construyeron categorías de temas que permitan expresar la información contenida en cada párrafo y finalmente estas categorías preliminares son contenidas en categorías generales, que agrupan una serie de temas específicos emergentes de los párrafos.

En la segunda etapa del análisis de contenido, se elabora el texto interpretativo provisional, en el cual se construyen inferencias, cuya finalidad es "hacer explícita en nuestra mente la información implícita en el pasaje" (León, 2003:24). De este modo, se organiza la información obtenida en torno a las categorías generales, diferenciando en el texto de investigación todos aquellos párrafos que corresponden a las categorías preliminares, así como también las observaciones establecidas en la observación de la densidad de la información, comentarios y notas al margen, etc. además de una revisión teórica que permita dar forma conceptual a las categorías levantadas.

La lectura a profundidad del texto, a través del análisis de contenido, presenta como resultado la emergencia de tres categorías pedagógicocurriculares referentes a: la Concepción de aprendizaje, el Sujeto de Aprendizaje y el Ejercicio de la Ciudadanía, las cuales muestran un nivel de intensidad y fuerza importante en la definición del Curriculum.

Para efecto de la presente investigación, solo se profundiza en la Concepción de Aprendizaje que subyace y configura el curriculum de adultos. La intención de profundizar en esta categoría pedagógico-curricular, permite identificar los valores, ideologías, supuestos y nociones que predominan y definen los conocimientos que se consideran como válidos y que están siendo aplicados en la educación de los jóvenes privados de libertad.

\section{Perspectiva legal de la educa- ción penitenciaria}

Primero, la educación como medida de corrección trae consigo una noción de tratamiento que releva la práctica rehabilitadora por encima del escenario de formación de valores. Al respecto, el Convenio 298 suscrito entre el Ministerio de Educación y el Ministerio de Justicia, expresa la importancia de realizar "una labor educativa conjunta, orientada a lograr la rehabilitación de los irregulares sociales y de conducta, internados en los establecimientos penales del País, que permitan su readaptación al medio social y su reincorporación a la vida activa y productiva de la nación" (Congreso Nacional de
Chile, 1978:3). Entre las medidas que se incorporan en este Convenio, se encuentra el promover la continuidad o mejora en los estudios y organizar una educación orientada a la condición particular de los reclusos, con proyección a establecer un subsistema educativo correccional.

En efecto, sobre el supuesto de que el joven es un sujeto que requiere corrección, la educación en el contexto privativo de libertad, se desarrollaría como una medida preventiva, cuyo propósito fundamental sería posibilitar su reinserción social (Ministerio de Justicia, 2005).

Bajo este supuesto de una educación como medida correctiva, la Política Educacional de Gendarmería (2006), expresaría dos líneas de acción en la educación de los adultos referentes a: formación para el trabajo y preocupación por las necesidades educativas de los internos, siendo éstas los factores más importantes de rehabilitación y reinserción social. "Esto no implica que deba prescindirse de la educación media científico humanista, pero si se ha de adoptar, ésta deberá complementarse en lo posible con algún tipo de capacitación laboral" (5).

El énfasis de formación para el trabajo, se considera como un pilar fundamental en la concepción de educación penitenciaria, destacando con ello que "los planes y programas de estudio penitenciarios deben dar énfasis en la toma de conciencia, acerca de la importancia de la formación para una vida de trabajo. Lo anterior, posibilita que el interno comprenda que el trabajo es un medio idóneo para dejar el delito" (Gendarmería de Chile Dirección Nacional, 2006:15).

En este sentido, se podría inferir que el acento de formación para el trabajo en los planes y programas de estudio, que plantea Gendarmería (2006), en el que no se releva la especialización o cualificación como meta de aprendizaje, pudiera remarcar las diferencias de ocupación entre una persona que sigue instrucciones y otra que es autónoma y dirige instrucciones.

En cuanto a las regulaciones existentes acerca de la implementación del curriculum de adultos en la educación de los jóvenes privados de libertad, es Gendarmería de Chile (2006) quien plantea dentro de sus normas técnicas de educación y cultura, la implementación del curriculum de adultos en la educación de los presos sin señalar que éste sea para los jóvenes o los menores de edad privados de libertad. "La oferta educacional penitenciaria se basa en los Planes y Programas de Educación de adultos del Ministerio de Educación, siendo aplicado en centros educativos al interior de los Establecimientos Penitenciarios del país, que consideran tanto escuelas 
básicas como también Liceos" (Ministerio de Justicia, 2008:8).

Por su parte, el Convenio 298 suscrito entre el Ministerio de educación Pública y el Ministerio de Justicia desde 1978, no explicita que sean los planes y programas de educación de adultos los que debiesen implementarse en la educación penitenciaria, solo señala dentro de los compromisos asumidos por el Ministerio de educación que éste debe, "aportar material didáctico para adultos, en uso por el Ministerio de Educación y el mobiliario que sea necesario para implementar los locales en forma subsidiaria a los que aporte el Ministerio de Justicia" (Congreso Nacional de Chile, 1978).

A su vez, ni la Ley General de Educación, ni el Marco curricular de educación de adultos (Ministerio de Educación, 2004) refieren que la educación penitenciaria se encuentre inserta en la modalidad de educación de adultos. Al respecto, la Ley General de Educación en su artículo 24 enuncia que: "la educación de adultos está dirigida a jóvenes y adultos que deseen iniciar o completar sus estudios" (Ministerio de Educación, 2009:8), sin especificar o describir los determinados contextos educacionales en los que se encuentra una población en particular, en este caso, los jóvenes privados de libertad.

Por otra parte, el Marco Curricular de Adultos tampoco especifica que dicha orientación sea aplicable o que regule la educación de los jóvenes que se encuentran en contextos privativos de libertad. En su artículo 7, expresa que: "se podrá autorizar la aplicación de planes y programas de estudio con una organización temporal y secuencial de Objetivos Fundamentales y Contenidos Mínimos Obligatorios diferente de la establecida en el presente decreto, en casos debidamente calificados y fundados, tales como, cuando el establecimiento atienda una población social y económicamente deprivada, o sometida a un régimen disciplinario especial" (Ministerio de Educación, 2004).

De esta manera, es posible observar que la implementación del curriculum de adultos en la educación de los jóvenes que se encuentran en contextos privativos de libertad, no posee una regulación o un fundamento legal que sostenga que esta modalidad sea la pertinente y que sea ésta la que debe aplicarse en la educación de los jóvenes en condición de reclusos.

\section{Categorías pedagógico- curriculares emergentes de la lectura a profundidad del currículum}

El curriculum de adultos sostiene una concepción de aprendizaje que promueve el desarrollo de competencias instrumentales para una efectiva inserción al ámbito laboral. Los hallazgos muestran la relevancia en el desarrollo de habilidades y actitudes instrumentales, e incorpora además un espacio curricular de formación instrumental, asociada con los ámbitos específicos de la vida de adultos: consumo y calidad de vida, inserción laboral, convivencia social y tecnologías de la información y la comunicación (Ministerio de Educación, 2004).

La evidencia en relación al desarrollo de competencias instrumentales, se muestra en los objetivos fundamentales transversales y a los sectores de aprendizaje presentes en la formación instrumental, en los cuales se enfatiza la importancia de desarrollar en los adultos aprendizajes como la perseverancia, la rigurosidad en el trabajo, la lealtad, el cumplimiento de normas y la capacidad de recibir consejos, contribuyendo con ello a la satisfacción personal y al sentido de vida y productividad (Ministerio de Educación, 2004).

En este sentido, ¿tiene el curriculum de adultos esta posibilidad de vivirse como una experiencia de construcción de conocimientos, habilidades, actitudes y valores que les permita a los distintos actores de la comunidad educativa cuestionarse, pensar y actuar críticamente en las estructuras sociales y culturales en las que está inserta la escuela? Al respecto, el curriculum esboza una serie de normas y disposiciones centradas en habilitar al adulto para la incorporación a la vida del trabajo, mostrando con mayor intensidad el carácter de instrumentalización y productividad en el aprendizaje como finalidad en la formación de los adultos.

Las evidencias encontradas, corroboran el carácter de instrumentalización del aprendizaje. Las unidades de registro correspondientes a los sectores y subsectores de aprendizaje de lenguaje y comunicación, idioma extranjero e inglés comunicativo, muestran la necesidad de desarrollar habilidades en la lectura y la escritura para: "participar activamente de la vida laboral, leer folletos instructivos y reglamentos, y escribir para completar formularios, elaborar informes o cartas. Se busca que como resultado de su paso por la educación básica, los adultos estén en condiciones de leer textos simples y funcionales y escribir en forma elemental" (Ministerio de Educación, 
2004:39).

No obstante, la concepción de un aprendizaje que promueve el desarrollo de competencias instrumentales no se inscribiría de manera tan intensa en el curriculum de educación regular básica y media, que está orientado a la educación de los niños, niñas y jóvenes (Ministerio de Educación, 2009). Las evidencias obtenidas en cuanto a las unidades de registro relacionadas con los requerimientos de diseño curricular, los principios valóricos del curriculum, las orientaciones en la selección curricular, la definición de la estructura curricular y los objetivos fundamentales transversales, muestran en la educación regular una visión de aprendizaje integrador que potencia la creatividad, iniciativa y crítica de los estudiantes.

A diferencia del curriculum de adultos, las competencias escolares en el curriculum dirigido a niños y jóvenes, se entienden como "sistemas de acción complejos que interrelacionan habilidades prácticas y cognitivas, conocimiento, motivación, orientaciones valóricas, actitudes y emociones que en conjunto se movilizan para realizar una acción efectiva" (67). Bajo esta premisa, las asignaturas o sectores de aprendizaje en el curriculum promueven la comprensión de la realidad, las habilidades cognitivas y procedimientos que posibilitan integrar y movilizar recursos, y las actitudes personales y éticas que orientan una acción responsable consigo mismo y con los demás.

Segundo, en torno al supuesto de un curriculum de adultos cuya concepción de aprendizaje es equivalente a la transmisión y adquisición de información, se presentan los resultados obtenidos a través de las unidades de registro relacionadas con los objetivos fundamentales transversales, los contenidos mínimos obligatorios y los sectores de aprendizaje de matemáticas, ciencias, estudios sociales e idioma extranjero, inglés.

Según el curriculum de adultos, la relevancia de la información responde al deber que tiene la persona de aportar a un mundo globalizado en el que el conocimiento crece de forma acelerada y en el cual se exige mayor dominio de habilidades, que le posibiliten aportar y actuar de manera efectiva en la sociedad (Ministerio de Educación, 2004).

No obstante, las evidencias obtenidas en relación a la noción de aprendizaje como equivalente a información, se muestran en los sectores de aprendizaje que presentan como indispensable el desarrollo de capacidades de organización, interpretación y uso de la información. Por ejemplo, la matemática en el curriculum de adultos se plantea como "un lenguaje especializado indispensable para lograr ma- yor capacidad en la organización de la información y describir las regularidades presentes en la realidad" (Ministerio de Educación, 2004:61).

Por otra parte, la asignatura de estudios sociales señala como prioridad en el aprendizaje entregar información a los adultos acerca de sus derechos y deberes, contribuyendo con ello a la comprensión de las características de la democracia. De igual modo, los objetivos fundamentales y los contenidos mínimos obligatorios del sector de aprendizaje de ciencias naturales, "se orientan en la formación de personas competentes en el uso de la información científica y tecnológica, de manera tal que los estudiantes puedan comprender su entorno de un modo distinto a como la habían construido desde su vida cotidiana" (Ministerio de Educación, 2004:85).

La representación del aprendizaje como información, sustentaría al curriculum de adultos en una racionalidad técnica que presentaría al conocimiento como objetivo y neutral, en la medida en que significa una realidad externa para el individuo de la cual se tiene que apropiar para desenvolverse exitosamente. En efecto, desde la racionalidad técnicainstrumental el aprendizaje se tiende a diseñar con fines medibles y concretos, reduciendo con ello la posibilidad de autoformación, en la medida en que no se desarrollaría como algo a ser cuestionado o analizado desde los aprendizajes y experiencias de vida previas. Esta pretensión de objetividad y neutralidad, avalaría la idea de que sean los grupos de expertos los que fijen los criterios normativos y los conocimientos que se consideran relevantes y necesarios en las vidas de las personas.

Tercero, con respecto a un curriculum cuyo énfasis adulto-centrista expresa un determinado modelo de adulto que se espera formar, el curriculum no señala explícitamente una noción de adulto como actor trabajador y productivo. Por lo tanto, es posible inferir que las características y experiencias cotidianas de las que se hace mención presentan a la base un conjunto de conocimientos, prácticas y valores de los adultos, asociados con el ámbito del trabajo, lo cual releva la importancia de "propiciar una práctica pedagógica que considere las experiencias previas de los alumnos, que los estimule a compartir vivencias de su vida laboral" (Ministerio de Educación, 2004:30)

$\mathrm{Al}$ respecto, las unidades de registro relacionadas con los requerimientos de diseño curricular, la definición de la estructura curricular, los ámbitos de formación, los objetivos fundamentales transversales y los sectores de aprendizaje de lenguaje, matemáticas e idioma extranjero, presentan la importancia de desarrollar la práctica educativa teniendo en cuenta las experiencias previas de los adultos. 
Lo que muestran las unidades de registro son experiencias de vida relacionadas con el ámbito laboral, en especial, las referidas a las competencias de empleabilidad de los adultos (Ministerio de Educación, 2004).

Por ejemplo, las experiencias previas puestas de relieve en el sector de aprendizaje lenguaje y comunicación, responden a que los adultos tienen bien establecido su lenguaje coloquial, en relación al ámbito laboral en el que se desenvuelven. Por ello, los aprendizajes de lenguaje darán importancia "a un incremento del vocabulario, un adecuado manejo en la construcción de oraciones y en el dominio de la ortografía en sus aspectos literales. Se prescinde de la enseñanza específica de la gramática como un contenido aislado de los problemas de comprensión y expresión de los estudiantes" (39).

Por otra parte, el sector de aprendizaje de educación matemática, señala la importancia de recurrir a la experiencia personal y cotidiana de los adultos para desarrollar contenidos significativos. Al respecto, explicita que "los estudiantes no son analfabetos matemáticamente; por el contrario durante su vida han pagado cuentas, medido y trozado maderas, telas $\mathrm{u}$ otros artículos en forma precisa, demostrando un manejo en aritmética y geometría que no se puede desconocer" (p.62). De lo anterior, se podría inferir que las experiencias de vida de un adulto, estarían determinadas por sus ocupaciones laborales, dejando de lado otra serie de conocimientos, valores, capacidades e intereses que pueden constituir su proyecto de vida.

No obstante, los Objetivos Fundamentales Transversales, los ámbitos de formación, los principios valóricos del curriculum y los sectores de aprendizaje de lenguaje, comunicación y educación matemática del curriculum de educación básica y media regular; expresan que la experiencia de aprendizaje debe atender las aptitudes, los intereses personales y las disposiciones vocacionales de los alumnos, de tal manera que sus decisiones y expectativas individuales - independientemente de sus opciones de egreso - puedan articularse con la cultura y el desarrollo social del país (Ministerio de Educación, 2009).

Por ejemplo, la orientación general de lenguaje y comunicación en el curriculum de educación básica y media regular, "promueve tomar conciencia del valor e importancia del lenguaje, la comunicación y la literatura para el crecimiento personal, la participación social y conocimiento, expresión y recreación del mundo interior y exterior" (Ministerio de Educación, 2009:31). Lo anterior, se posibilita cuando se estimula la creatividad e iniciativa de los estudiantes y se potencian sus habilidades de expresión e interacción social.

Asimismo, la propuesta formativa en este sector de aprendizaje se orienta al desarrollo del pensamiento crítico, reflexivo y la capacidad de diálogo. Esto implica enriquecer el lenguaje con el que los estudiantes ingresan al sistema escolar, incorporando sus diversas realidades socio-culturales, con el propósito de estimularlos para pensar, crear, recrearse y desarrollar la identidad en una dimensión personal y social.

Por otro lado, los resultados obtenidos en las unidades de registro correspondientes al sector de educación matemática, muestran un aprendizaje que promueve la comprensión de la realidad y la autonomía en el estudiante, permitiéndole con ello el desarrollo de la confianza en sus propias capacidades. Los ejes que articulan la experiencia formativa en el sector, hacen énfasis en "el desarrollo del pensamiento creativo y crítico, favoreciendo la comprensión por sobre el aprendizaje de reglas y mecanismos sin sentido, pues apela al conocimiento como una creación culturalmente situada como potencial para aumentar la capacidad del ser humano de comprender e intervenir en el medio que le rodea" (Ministerio de Educación, 2009:148)

En el curriculum de educación básica y media regular, se aprecia un espacio para la exploración, la indagación, la experimentación, la inventiva y la posibilidad que los estudiantes potencien y fortalezcan sus capacidades, conocimientos y experiencias con el contexto. La finalidad en la educación está planteada desde el desarrollo de una identidad formativa que le posibilite a los niños y jóvenes potenciar sus capacidades, habilidades, valores y conocimientos fundamentales para su crecimiento personal y desenvolvimiento en la sociedad (Ministerio de Educación, 2009:2009).

En cuanto al curriculum de adultos, se infiere una noción de sujeto de aprendizaje cuya historia, proyecciones y expectativas, no estarían planteadas como fines del proceso educativo, pues lo que se releva como experiencia vital, estaría asociado con el ámbito laboral cuasi-calificado. Este modelo de formación, tiende a mostrar a una persona cuyo proyecto de vida estaría forjado en función de obtener un trabajo acorde a sus habilidades instrumentales, por lo cual el aprendizaje no sería vivenciado como un valor y fin en sí mismo, sino meramente como medio para sostener una posición de trabajador. 


\section{Conclusiones}

\section{¿Por qué el marco curricular de adul- tos, es la orientación que regula la educación de los jóvenes que se en- cuentran en contextos privativos de li- bertad?}

Los resultados muestran que la decisión de implementar el curriculum de adultos en la educación de los jóvenes que se encuentran privados de libertad, no está sustentada en las leyes y políticas educativas que regulan la educación Chilena.

Ni la Ley General de Educación (Ministerio de Educación, 2009):, ni el marco curricular de educación de adultos (Ministerio de Educación, 2004), señalan que el curriculum de adultos sea el marco que regule la educación en contextos privativos de libertad para menores de edad. Además, la Ley y la modalidad curricular, tampoco explicitan dentro de sus fines y objetivos atender a los jóvenes que se encuentran insertos en este tipo de contexto.

Por otra parte, quien sí explicita la implementación de los planes y programas de adultos en la educación penitenciaria, es Gendarmería de Chile (2006). Desde su política educacional, considera como pilar fundamental la formación para el trabajo y la atención a las necesidades educativas de los internos. Ambos pilares se enfatizan en las finalidades y objetivos del curriculum de adultos (Ministerio de Educación, 2004).

Ésta política educacional, parte de las consideraciones estipuladas desde hace más de 30 años en el Convenio 298 suscrito por el Ministerio de Educación Pública y el de Justicia, el cual aprueba "una labor educativa conjunta orientada a lograr la rehabilitación de los irregulares sociales y conductuales que se encuentran en establecimientos penitenciarios" (Congreso Nacional de Chile, 1978:3)

Este Convenio, releva el lugar fundamental que tiene la educación en la readaptación del recluso a la sociedad y en su reincorporación a la vida activa y productiva del país. De allí que los pilares de la educación para Gendarmería, posean una noción de corrección que incide en que el recluso se rehabilite y se reinserte en la sociedad (Gendarmería de Chile Dirección Nacional, 2006).

Bajo el panorama anterior, se logra apreciar que tanto la Ley como la Política educativa, expresan un silencio, en cuanto a la regulación del proceso educativo de los jóvenes que se encuentran en contextos privativos de libertad. Lo anterior, conlleva a plantear los siguientes interrogantes:
¿Conoce el Ministerio de Educación la realidad educativa de los jóvenes que están privados de libertad?, ¿Interesa a la Política Educativa, construir una orientación pedagógico-curricular, basada en las expectativas, intereses y necesidades de la población juvenil recluida en contextos de privación de libertad? y ¿Este silencio devela acaso, un desinterés y una despreocupación por la realidad socioeducativa de los jóvenes que se encuentran privados de libertad?

Por lo anterior, la consecuencia de este silencio pudiera estar asociada con una reproducción no reflexiva, de las decisiones pedagógicas-curriculares tomadas hace más de treinta años en relación a una educación para la rehabilitación y corrección de quienes se encontraban en contextos penitenciarios. Ésta reproducción, no solo desconoce la realidad educativa de los jóvenes que están privados de libertad, sino que además no muestra un interés suficiente por reestructurar un educación y orientación curricular pertinente para una población con características específicas como las que tiene ésta.

\section{¿Cuál es el propósito del curriculum que se implementa en la educación de los jóvenes privados de libertad?}

Los resultados obtenidos presentan un curriculum de adultos que supone una concepción de aprendizaje, basada en la promoción y desarrollo de habilidades instrumentales para una efectiva inserción laboral y productiva, lo cual permitiría a los jóvenes proyectarse una vida distinta al delito.

Una de las consecuencias de esta lógica instrumental del curriculum para la inserción laboral, se manifiesta en la omisión de las realidades particulares y condiciones de vida socio-culturales de los jóvenes que se encuentran en este contexto. El curriculum bajo esta lógica técnico-instrumental, excluye las experiencias, proyecciones, capacidades individuales y la historia de vida de éstos jóvenes.

De esta manera, más que un aprendizaje cuya construcción social posibilita la negociación colectiva y participativa de intereses, necesidades y proyecciones de los jóvenes que se encuentran recluidos, hay un sentido de funcionalidad donde el aprendizaje como medio de producción se alinea con las demandas y exigencias del mundo laboral y productivo.

Por otra parte, la priorización y caracterización de conocimientos, habilidades, actitudes y valores basados en las relaciones sociales de subordinación, como aprender a recibir instrucciones o a desarrollar eficazmente una tarea laboral, aseguran la co- 
rrespondencia con las jerarquías del puesto de trabajo. Lo anterior, complejiza la autoformación y el desarrollo de una práctica emancipadora.

Asimismo, bajo la perspectiva instrumental del curriculum, el conocimiento se presenta como neutral y objetivo, reduciendo toda posibilidad de los actores educativos en crear, innovar y construir un conocimiento intersubjetivo. De hecho, desde esta mirada, son los expertos y las autoridades educativas, las personas idóneas en diseñar y fijar un marco de conocimiento y valores, que se constituirán como metas indispensables en el buen funcionamiento de la sociedad.

Lo anterior, ignora la participación de los profesores, profesionales y jóvenes en la construcción de un proyecto educativo democrático pertinente con sus proyectos de vida, sus necesidades e intereses, vinculándolos con la realidad del contexto sociocultural, económico y político, en el que se encuentran insertos, de modo que la propuesta curricular no sea sobre los actores educativos, sino con los actores educativos.

Por último, la instrumentalización del curriculum escolar, presenta el conocimiento a los jóvenes, como algo que no puede ser cuestionado o analizado desde sus aprendizajes previos y experiencias de vida, perdiéndose con ello, la dimensión subjetiva del conocer y el reconocimiento de su historia de vida.

\section{¿Constituye la propuesta curricular más pertinente para la educación de los jóvenes privados de libertad?}

Los resultados obtenidos expresan que el curriculum de adultos supone una concepción de aprendizaje que no es acorde a la educación de los jóvenes, puesto que rescata un modelo de adulto trabajador, que ignora la trayectoria vital de los jóvenes.

El curriculum como extensión del escenario laboral, potencia la heteronomía de los individuos, lo cual se traduce, en este caso, en la modelación de un sujeto de aprendizaje como trabajador, cuyos valores, actitudes y conocimientos fundamentales a potenciar son la obediencia a la norma, el seguimiento de instrucciones, la puntualidad y constancia (Ministerio de Educación, 2004). Estos valores, actitudes y conocimientos que plantean el sujeto de aprendizaje que se pretende formar en el curriculum de adultos, terminan asegurando las relaciones sociales, basadas en la desigualdad.

El impacto de la heteronomía se traduce en la incapacidad que tiene el sujeto de aprendizaje en reconocer la iniciativa humana, los conocimientos que se han desarrollado a lo largo de su trayectoria y su experiencia de vida, aspecto que complejiza el desarrollo de una libertad individual, que para el caso específico de los jóvenes que se encuentran recluidos, les impide consolidar una postura propia, enmarcada en la responsabilidad social consigo mismos y con los demás actores de la sociedad.

En conclusión, el curriculum de adultos presenta una visión de sujeto de aprendizaje, que no corresponde al sujeto real que se encuentra en el contexto en el cual se implementa. Además está pensado para ser utilizado en una población con una experiencia y una etapa de desarrollo distinto a los jóvenes que se encuentran privados de su libertad.

\section{Desafíos y recomendaciones de política pública}

Se recomienda que la legislación actual en materia de educación explicite los fines y objetivos de la educación de los y las jóvenes que se encuentran en un contexto privativo de libertad.

Debe existir una articulación entre el Ministerio de Justicia y Ministerio de Educación, respecto de las orientaciones socio-educativas y pedagógicascurriculares, que sustentan la formación de los y las jóvenes que se educan en un contexto privativo de libertad.

Es esencial la construcción de una propuesta curricular, basada en la concepción de formación de un sujeto activo de Derechos en la que se incluye la participación de los distintos actores educativos, jóvenes, docentes, directivos, funcionarios del Servicio Nacional de Menores, familias, Defensoría, Consejo Nacional de la Infancia, etc.

De esta manera, se recomienda revisar de manera exhaustiva la concepción e implementación de la Ley de Responsabilidad Penal Adolescente, en cuanto a la noción de "intervención socio-educativa amplia", en la que no se define o señala las orientaciones pedagógicas-curriculares que serían favorables desarrollar en el marco de un contexto privativo de libertad y que pueden contribuir a generar escenarios de formación y ejercicio de los valores o principios fundamentales.

Por último, se recomienda incluir en la propuesta curricular participativa, los instrumentos legales internacionales que sustenta los procesos de prevención, formación y protección para los jóvenes privados de libertad, entre los que figuran, la Convención de los Derechos del Niño, las Directrices Riad de las Naciones Unidas para la prevención de la delincuen- 
cia juvenil y las normas mínimas aceptadas por las Naciones Unidas sobre la protección de los menores de edad privados de libertad. Contribuyendo con esto a la implementación de una efectiva estrategia de protección y garantía al derecho de una educación pertinente y de calidad.

\section{Referencias}

Aguirrezabal Grünstein, M., Lagos Carrasco, G., y Vargas Pinto, T. (2009). Responsabilidad penal juvenil: hacia una "justicia individualizada". Revista de derecho, 22(2):137-159.

Apple, M. W. (1986). Ideología y currículo. Ediciones Akal, Madrid.

Bericat, E. (1998). La integración de los métodos cuantitativo y cualitativo en la investigación social: Significado y medida. Ariel, Barcelona.

Bowles, S. y Gintis, H. (1976). Schooling in capitalist America. Basic Books, Nueva York.

Congreso Nacional de Chile (1978). Convenio 298: entre Ministerio de Educación Pública y Ministerio de Justicia. Santiago.

Couso, J. (2007). Principio educativo y (re) socialización en el Derecho penal juvenil. Justicia y Derechos del Niño, 9.

Da Silva, T. T. (1999). Documentos de identidad. Una introducción a las teorías del curriculum. Auténtica, Belo Horizonte.

Flick, U. (2004). Introducción a la investigación cualitativa. Fundación Paideia Galiza, A Coruña.

Foucault, M. (2009). Vigilar y castigar: nacimiento de la prisión. Siglo Veintiuno, México D. F.

Gendarmería de Chile Dirección Nacional (2006). Estudio de la Educación Penitenciaria del Departamento de Readaptación de Gendarmería de Chile. Revista Estudios Penitenciarios.
Giroux, H. A. (1990). Los profesores como intelectuales: hacia una pedagogía crítica del aprendizaje. Barcelona Paidós, Barcelona.

León, J. A., editor (2003). Conocimiento y discurso: claves para inferir y comprender. Madrid : Eds. Pirámide, Madrid.

Ministerio de Educación (2004). Marco Curricular de la Educación Básica y Media de Adultos. Objetivos Fundamentales y Contenidos Mínimos Obligatorios. Santiago.

Ministerio de Educación (2009). Ley General de Educación.

Ministerio de Justicia (2005). Ley número 20.084.

Ministerio de Justicia (2008). Normas técnicas en educación y cultura penitenciaria. Santiago.

Organización de las Naciones Unidas (2007). Recopilación de reglas y normas de las Naciones Unidas en la esfera de la prevención del delito y la justicia penal. Nueva York.

Ruiz Olabuénaga, J. I. (2003). Metodología de la investigación cualitativa. Universidad de Deusto, Bilbao.

Sandín, E. (2003). Investigación cualitativa en educación. fundamentos y tradiciones. McGraw-Hill, Madrid.

UNICEF (1996). Convención sobre los Derechos del Niño: aprobada por la Asamblea General de las Naciones Unidas el 20 de noviembre de 1989. UNICEF Chile, Santiago.

Valenzuela, J. (2009). La pena y la educación. Una aproximación al fundamento de la pena juvenil. Revista de Estudios de la Justicia, 11.

Valles, M. S. (2000). Técnicas cualitativas de investigación social. Sintesis Editorial, Madrid. 